\title{
Percutaneous release of the plantar fascia. New surgical procedure
}

\author{
Francesco Oliva ${ }^{1}$ \\ Eleonora Piccirilli ${ }^{1}$ \\ Umberto Tarantino ${ }^{1}$ \\ Nicola Maffulli2
}

1 Department of Orthopaedics and Traumatology, University of Rome "Tor Vergata", Italy

2 Department of Musculoskeletal Disorders, University of Salerno, Salerno; Campus Bio-Medico University of Rome, Rome, Italy; Centre for Sports and Exercise Medicine, Barts and The London School of Medicine and Dentistry, Mile End Hospital, London, UK

\author{
Corresponding author: \\ Francesco Oliva \\ Department of Orthopaedics and Traumatology, \\ University of Rome "Tor Vergata" \\ Viale Oxford 81 \\ 00133 Rome, Italy \\ Email: olivafrancesco@hotmail.com
}

\section{Summary}

Background: Plantar fasciopathy presents with pain at the plantar and medial aspect of the heel. If chronic, it can negatively impact on quality of life. Plantar fasciopathy is not always self-limiting, and can be debilitating.

Methods: Surgical management involves different procedures. We describe a percutaneous plantar fascia release. A minimally invasive access to the plantar tuberosity of the calcaneus is performed, and a small scalpel blade is used to release the fascia.

Conclusion: With this procedure, skin healing problems, nerve injuries, infection and prolonged recovery time are minimised, allowing early return to normal activities.

Level of Evidence: V.

KEY WORDS: plantar fasciopathy, plantar fascia release, mininvasive release, percoutaneous surgery.

\section{Background}

Patients with plantar fasciopathy report sharp pain in the plantar aspect of the foot, in the medial portion of the heel ${ }^{1}$. Both sedentary patients and athletes ${ }^{2}$ suffer from it, with a higher frequency between the age of 40 and $60^{3}$. The plantar fascia, a fibrous band of connective tissue (aponeurosis), helps to maintain the stability and the arch of the foot ${ }^{4}$. In plantar fasciopathy there is non-inflammatory structural breakdown of the plantar fascia rather than an inflammatory process ${ }^{5}$, secondary to myxoid degeneration with microtears within the plantar fascia, collagen necrosis and angiofibroblastic hyperplasia of $i^{6}$. The diagnosis is essentially clinical. Plain radiography has limited value, while ultrasonography is an useful, non-invasive, well-tolerated and reliable tool ${ }^{7}$. Plantar fasciopathy is not always self-limiting. Conservative management involves activity modifications, stretching $^{7-10}$ of the plantar fascia, ice massage, and night dorsiflexion splints or orthotics. If patients do not experience a resolution of symptoms, the use of nonsteroidal anti-inflammatory drugs (NSAIDs) is common. Steroid injections can be performed for a short term pain relief ${ }^{7,11}$. There is no clear evidence on the efficacy of platelet-rich plasma (PRP) injections in chronic cases ${ }^{7,12}$. Focal extracorporeal shockwave therapy can be used in patients with recalcitrant plantar fasciopathy after non-invasive treatments have failed $^{13}$, with success rates between 50 and $65 \%{ }^{14}$ and the possibility for the patients to remain active during treatment ${ }^{15}$. Manual stretching exercises specific to the plantar fascia in combination with repetitive low-energy radial shock-wave therapy can be of help ${ }^{16}$. If the patient continues to have symptoms which do not respond to conservative management for more than 6-12 months, more invasive measures or surgery should be contemplated. Several procedures have been described: open plantar fascia release, endoscopic plantar fasciotomy through a medial portal ${ }^{17}$ that can be associated to ultrasound assistance ${ }^{18}$, percutaneous or open plantar fasciotomy with heel spur resection ${ }^{19}$, neurectomy or neurolysis of the medial calcaneal nerve, gastrocnemius reces$\operatorname{sion}^{20}$. Calcaneal osteotomy has been associated to prevent lateral column pain from the loss of the longitudinal arch height after surgery ${ }^{21}$. We describe a minimally invasive technique for percutaneous plantar fascia release.

\section{Surgical procedure}

Under general or regional anesthesia, the patient is supine, and fluoroscopy is used to identify the calcaneal tuberosity and the correct site of section of the origin of plantar fascia (Fig. 1). A stab wound is pro- 


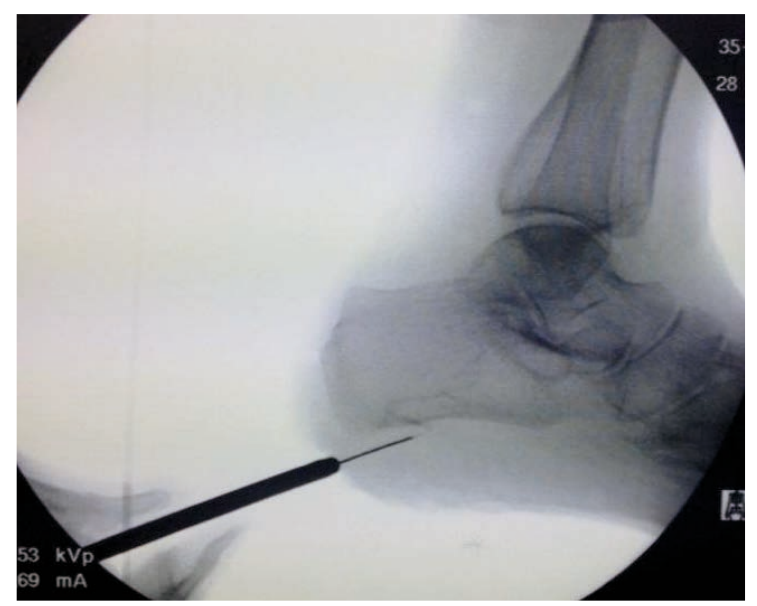

Figure 1. Identification of the calcaneal tuberosity and the correct site of insertion at the origin of plantar fascia.

duced over the plantar aspect of the foot, to reach the plantar tuberosity of the calcaneus (Fig. 2). A section of the medial $1 / 3$ of the plantar fascia from its calcaneal attachment is performed. If a calcaneal spur is present, a cylindrical bur with four sectors $\varnothing 4.1 \mathrm{~mm}$ (produced by Bone) is inserted in the wound, and advanced towards the plantar aspect of the calcaneus. The lateral portion of the plantar fascia is then identified by moving the bur medially and laterally, until the surgeon experiences the resistance of the plantar fascia (Fig. 3). The calcanear spur is then resected under image intensifier control. At the end of the procedure, the bur is removed, abundant wash out is performed with normosaline, and the wound is closed with a non absorbable monofilament suture (Fig. 4). Weight bearing as able is allowed from the day of the surgery, protecting the foot with a bulky bandage for two weeks, during which the patient performs mobi-

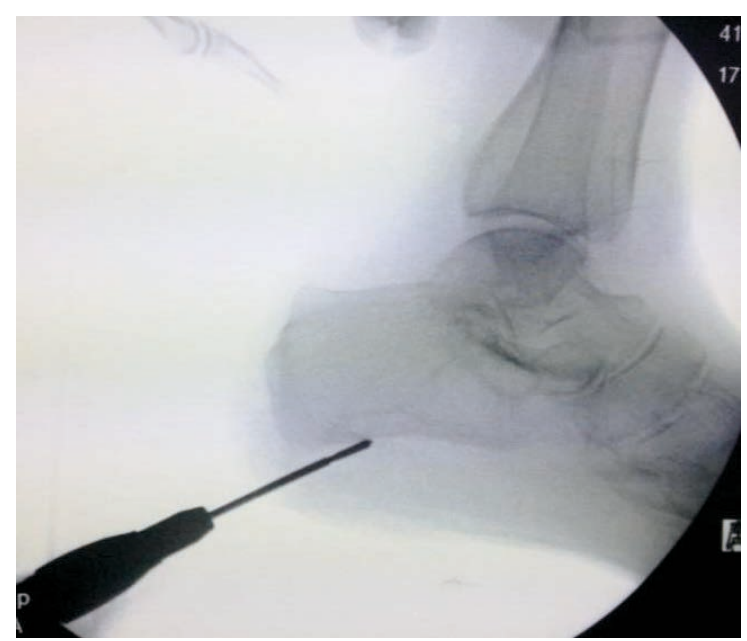

Figure 2. Insertion of the bur to reach the plantar tuberosity of the calcaneus.

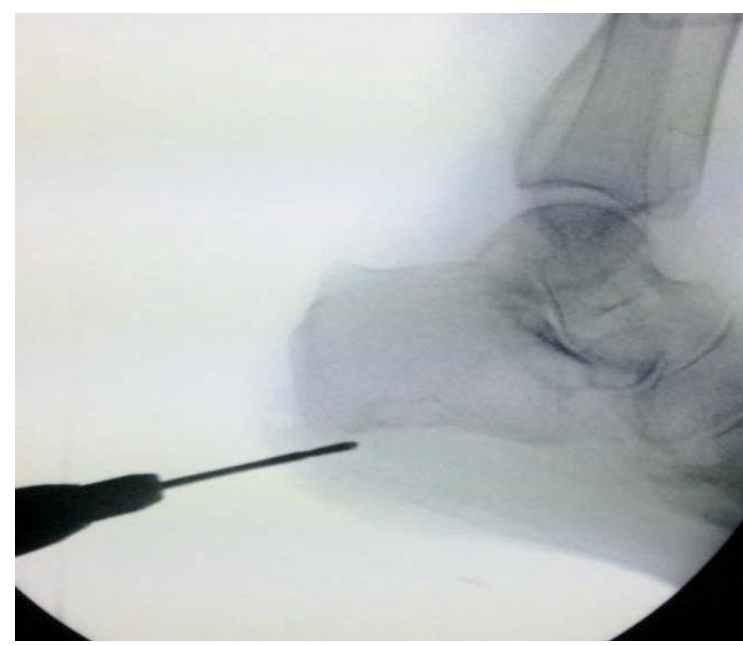

Figure 3. The bur is moved medially and laterally to identify the plantar fascia.

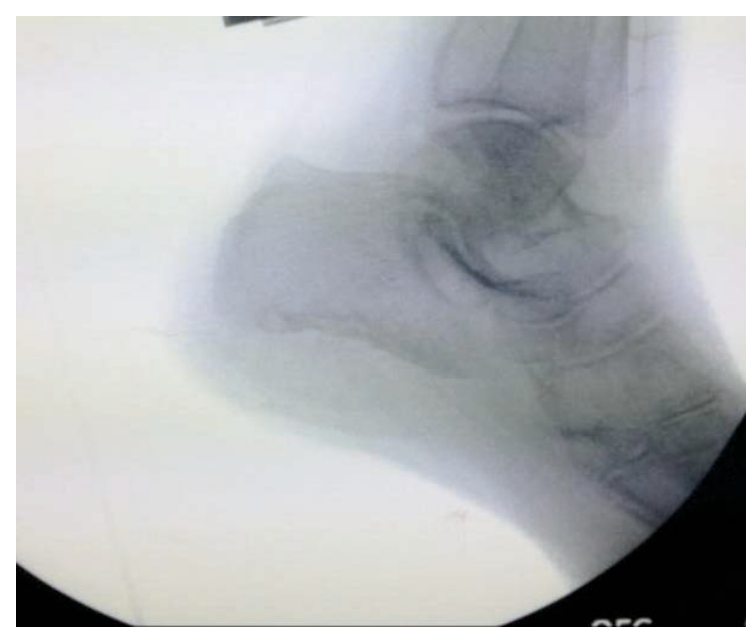

Figure 4. The bur is removed at the end of the procedure.

lization of the toes, ankle and foot. After removal of the bandage, formal rehabilitation is started. Over the next four weeks, patients can gradually return to their normal activities. A gradual return to sports is allowed, avoiding those that involve repetitive impact, such as running (even on a treadmill), for three months.

\section{Discussion}

Plantar fasciopathy is a common cause of foot pain, often resistant to conservative management. Different surgical techniques have been described to manage plantar fasciopathy ${ }^{22}$. Many of these techniques impact negatively on the anatomy of the soft tissues of the plantar aspect of the foot, and may alter the foot biomechanics and its ability to balance the stress of weight bearing. In addition, total plantar fasciotomy may result in changes to the pressure zones of the foot and it could lead to a secondary collapsed arch 
with persistent severe pain after surgery ${ }^{23}$. The percutaneous technique described in the present article releases the medial one third of the plantar fascia through a single posterior plantar portal. In this way, skin healing problems, nerve disturbance, infection and persistent pain associated with prolonged recovery time are avoided. This minimal release impacts favourably with pain, allowing an early return to normal activities ${ }^{24}$.

\section{Conflict of interest}

The Authors have no conflict of interest.

\section{References}

1. Melvin TJ, Tankersley ZJ, Qazi ZN, Jasko JJ, Odono R, Shuler FD. Primary Care Management of Plantar Fasciitis. W V Med J. 2015;111(6):28-32.

2. Sammarco GJ, Helfrey RB. Surgical treatment of recalcitrant plantar fasciitis. Foot Ankle Int. 1996;17:520-526.

3. Langford CA, Gilliland BC. Plantar fasciitis. In: Longo DL, Fauci AS, Kasper DL, et al, eds. Harrison's Principles of Internal Medicine. 18th ed. New York. NY: McGraw-Hill. 2012, chap 337.

4. Hormozi J, Lee S, Hong DK. Minimal Invasive Percutaneous Bipolar Radiofrequency for Plantar Fasciotomy: A Retrospective Study. The Journal of Foot and Ankle Surgery. 2011; 50:283-286.

5. Yin MC, Ye J, Yao M, et al. Is extracorporeal shock wave therapy clinical efficacy for relief of chronic, recalcitrant plantar fasciitis? A systematic review and meta-analysis of randomized placebo or active-treatment controlled trials. Arch Phys Med Rehabil. 2014;95(8):1585-1593.

6. Lemont H, Ammirati KM, Usen N. Plantar fasciitis: a degenerative process (fasciosis) without inflammation. J Am Podiatr Med Assoc. 2003;93:234-237.

7. Tee Lim A, How How C, Tan B. Management of plantar fasciitis in the outpatient setting. Singapore Med J. 2016;57(4):168171.

8. Digiovanni BF, Nawoczenski DA, Malay DP, et al. Plantar fascia-specific stretching exercise improves outcomes in patients with chronic plantar fasciitis. A prospective clinical trial with two-year follow-up. J Bone Joint Surg Am. 2006;88:17751781.

9. Rompe JD, Cacchio A, Weil L Jr, et al. Plantar fascia-specific stretching versus radial shock-wave therapy as initial treatment of plantar fasciopathy. J Bone Joint Surg Am. 2010;92 (15):2514-2522.
10. Huffer D, Hing W, Newton R, Clair M. Strength training for plantar fasciitis and the intrinsic foot musculature: A systematic review. Phys Ther Sport. 2016.

11. Moustafa AM, Hassanein E, Foti C. Objective assessment of corticosteroid effect in plantar fasciitis: additional utility of ultrasound. Muscles Ligaments Tendons J. 2016;5(4):289-296.

12. Franceschi F, Papalia R, Franceschetti E, Paciotti M, Maffulli $\mathrm{N}$, Denaro V. Platelet-rich plasma injections for chronic plantar fasciopathy: a systematic review. Br Med Bull. 2014;112 (1): 83-95.

13. Rompe JD, Furia J, Weil L, Maffulli N. Shock wave therapy for chronic plantar fasciopathy. Br Med Bull. 2007;81-82:183-208.

14. Gollwitzer H, Saxena A, Di Domenico L, et al. Clinically relevant effectiveness of focused extracorporeal shock wave therapy in the treatment of chronicplantar fasciitis: a randomized, controlled multicenter study. J Bone Joint Surg Am. 2015;97 (9):701-708.

15. Saxena A, Fournier M, Gerdesmeyer L, Gollwitzer H. Comparison between extracorporeal shockwave therapy, placebo ESWT and endoscopic plantar fasciotomy for the treatment of chronic plantar heel pain in the athlete Muscles Ligaments Tendons J. 2013;2(4):312-316.

16. Rompe JD, Furia J, Cacchio A, Schmitz C, Maffulli N. Radial shock wave treatment alone is less efficient than radial shock wave treatment combined with tissue-specific plantar fasciastretching in patients with chronic plantar heel pain. Int J Surg. 2015;24(Pt B):135-142.

17. Saxena A. Uniportal endoscopic plantar fasciotomy: a prospective study on athletic patients. Foot Ankle Int. 2004;25 (12):882-889.

18. Ohuchi H, Ichikawa K, Shinga K, Hattori S, Yamada S, Takahashi K. Ultrasound-assisted endoscopic partial plantar fascia release. Arthrosc Tech. 2013;2(3):e227-230.

19. Fallat LM1, Cox JT, Chahal R, Morrison P, Kish J. A retrospective comparison of percutaneous plantar fasciotomy and open plantar fasciotomy with heel spur resection. J Foot Ankle Surg. 2013;52(3):288-290.

20. Cychosz CC, Phisitkul P, Belatti DA, Glazebrook MA, Di Giovanni $\mathrm{CW}$. Gastrocnemius recession for foot and ankle conditions in adults: Evidence-based recommendations. Foot Ankle Surg. 2015;21(2):77-85.

21. Miyamoto W, Takao M, Uchio Y. Calcaneal osteotomy for the treatment of plantar fasciitis. Arch Orthop Trauma Surg. 2010;130(2):151-154.

22. Neufeld SK, Cerrato R. Plantar Fasciitis: evaluation and treatment. J Am Acad Orthop Surg. 2008;16:338-346.

23. Assad S, Ahmad A, Kiani I, Ghani U, Wadhera V, Tom TN Novel and Conservative Approaches Towards Effective Management of Plantar Fasciitis. Cureus. 2016;8(12):e913.

24. Padulo J, Oliva F, Frizziero A, Maffulli N. Muscles, Ligaments and Tendons Journal - Basic principles and recommendations in clinical and field science research: 2016 update. MLTJ. 2016;6(1):1-5. 